\title{
Infant respiratory infections and later respiratory hospitalisation in childhood
}

\author{
Hannah C. Moore ${ }^{1}$, Graham L. Hall ${ }^{2}$ and Nicholas de Klerk ${ }^{3}$ \\ Affiliations: 'Wesfarmers Centre of Vaccines and Infectious Diseases, Telethon Kids Institute, The University of \\ Western Australia, Perth, Australia. ${ }^{2}$ Paediatric Respiratory Physiology, Telethon Kids Institute, The University \\ of Western Australia, Perth, Australia. ${ }^{3}$ Centre for Biostatistics, Telethon Kids Institute, The University of \\ Western Australia, Perth, Australia.
}

Correspondence: Hannah C. Moore, Wesfarmers Centre of Vaccines and Infectious Diseases, Telethon Kids Institute, The University of Western Australia, PO Box 855, West Perth, WA, Australia 6872.

E-mail: hannah.mooreatelethonkids.org.au

ABSTRACT Acute respiratory infections (ARI) cause significant morbidity in infancy. We sought to quantify the relationship between ARI and development of respiratory morbidity in early childhood.

Population-based longitudinal hospitalisation data were linked to perinatal, birth and death records for 145580 Western Australian children from 1997 to 2002. We conducted Cox regression with sensitivity analyses to quantify the risk of recurrent ARI in infancy for respiratory hospitalisation after the age of 3 years.

ARI in infancy was significantly related to respiratory hospitalisation before (hazard ratio (HR) 3.5, 95\% CI 3.1-3.8) and after (HR 3.0, 95\% CI 2.6-3.4) adjusting for known risk factors including maternal smoking during pregnancy, season of birth, delivery mode and gestational age. We noted a dose response with the number and length of infant ARI hospitalisations and increasing risk with no effect modification by gestational age. Results were similar when later respiratory hospitalisations were restricted to asthma hospitalisations only.

Recurrent hospitalisations for ARI in infancy significantly increase the risk of respiratory morbidity and asthma requiring hospitalisation after the age of 3 years in a dose-response fashion. The increase in relative risk is not modified by gestational age. Efforts to reduce the occurrence of infant ARI are likely to have significant public health benefits.

@ERSpublications

Recurrent infant acute respiratory infections: the major predictor of later hospitalisation for respiratory morbidity http://ow.ly/NVmGv

\footnotetext{
This article has supplementary material available from erj.ersjournals.com

Received: Nov 122014 | Accepted after revision: May 162015 | First published online: Aug 202015

Support statement: This work was supported by National Health and Medical Research Council (NHMRC) project grant APP572590. H.C. Moore is supported by NHMRC Early Career Fellowship APP1034254. G. Hall holds a NHMRC Fellowship APP1025550. Funding information for this article has been deposited with FundRef.
}

Conflict of interest: None declared.

Copyright OERS 2015 


\section{Introduction}

Acute respiratory infections (ARI) such as bronchiolitis, whooping cough, pneumonia and influenza are a major cause of morbidity in young children, with hospitalisation rates in industrialised countries ranging from 31-63/1000 in non-Indigenous children [1,2] to 116-427/1000 in Indigenous children [2, 3]. Those at highest risk of respiratory infections include boys, autumn-born infants, those born prematurely, low socioeconomic status, those whose mothers smoked during pregnancy and those with birth defects such as congenital heart disease and chronic lung disease $[4,5]$.

Numerous studies have identified associations between early ARI and subsequent asthma [6-11], but, with the exception of an analysis of a population cohort of Danish twins [10] these have been smaller prospective studies of 140-259 patients recruited and prospectively followed, with $55-100 \%$ having a family history of atopy and/or asthma. There has also been debate whether the relationship between ARI and asthma is causal or purely association, and it has been suggested that the severity of early ARI may have a part to play in the development of asthma [12]. In addition, some of the risk factors for asthma and later respiratory morbidity are the same as the risk factors for ARI, such as Caesarean delivery [13-15] and preterm birth $[16,17]$. Large total-population based studies on otherwise healthy populations adjusting for known risk factors may improve our understanding of these relationships through the conduct of subgroup and sensitivity analyses. Linked administrative data provides this opportunity [18]

The aim of this study was to use total-population based data on an otherwise healthy population of children to assess the relationship between early ARI requiring hospitalisation and subsequent hospitalisation for respiratory causes including asthma, and investigate any effect modification with other known risk factors for respiratory morbidity.

\section{Methods}

Setting and data sources

The Western Australian Data Linkage System (WADLS) is a unique system that brings together data from numerous administrative and health datasets, many of which have $100 \%$ coverage of data on the total population [18]. Using the WADLS, we designed a retrospective cohort study of 245249 singleton live births in Western Australia between 1996 and 2005. Full details of data cleaning are found elsewhere [4, 19]. In brief, our study consisted of longitudinal data from the Midwives Notification System, Birth Register, Death Register, Hospital Morbidity Database System and the Western Australian Register for Developmental Anomalies.

These datasets contain information on maternal and infant perinatal factors such as age, sex, birthweight, gestational age, pregnancy and labour complications, Aboriginal status of the mother and infant and some maternal conditions including asthma and smoking during pregnancy. Percentage of optimal birthweight, a measure taking into account gestational duration, fetal sex, maternal age, maternal height and parity [20] was used as a measure of fetal growth and appropriateness of fetal growth and grouped into three categories (low $<85 \%$, normal $85-114 \%$ or high $\geqslant 115 \%$ ). Socioeconomic status was derived from the Socio-Economic Index for Areas (SEIFA) [21], using postcode of residence at the time of birth. SEIFA is comprised of several indices, the main index being that of relative disadvantage, which is derived from low income, low educational attainment, high unemployment and jobs in unskilled occupations. SEIFA scores are measured at the collection district level, the smallest unit available for population-based analyses, and grouped into quantiles. Birth defects recorded on the Western Australia Register for Developmental Anomalies were defined as the presence of one or more structural or functional anomalies that are present at conception or occur before the end of pregnancy and diagnosed by 6 years of age [22]. The use of these population-based linked data was approved by the Western Australian Department of Health Human Research Ethics Committee and the Western Australian Aboriginal Health Ethics Committee.

\section{Hospitalisation measures}

International Classification of Diseases, 10th revision (ICD-10) diagnosis codes were used to identify hospitalisations of interest. Early ARI was defined as hospitalisation in the first year of life with one of the following diagnoses listed in either the principal or 20 additional diagnosis codes: acute bronchiolitis (J21), pneumonia (J12-J18, B59, B05.2, B37.1 or B01.2), influenza (J10 or J11), whooping cough (A37), acute bronchitis (J20), unspecified acute lower respiratory infection (J22), asthma (J45) and symptom of wheeze (R06.2). Asthma diagnosis codes were included to allow for likely misclassification of ARI in the first year of life. The primary outcome variable was hospitalisation for asthma after the age of 3 years. However, in order to account for diagnostic shifts between bronchiolitis and asthma, as reported in this population [23] and elsewhere $[24,25]$, a respiratory morbidity indicator was developed that included any mention of the following ICD-10 diagnoses in either the principal or additional diagnosis fields: asthma (J45), acute bronchiolitis ( J21), unspecified acute lower respiratory infection ( J22) and symptom of wheeze (R06.2). We 
assessed any changes in the composition of the ARI and respiratory hospitalisation categories over time. To test the robustness of these categories, we conducted sensitivity analyses. We restricted our exposure variable, ARI, to only hospitalisations in infancy coded for either bronchiolitis, pneumonia, influenza, whooping cough, bronchitis and unspecified acute lower respiratory infection and restricted our outcome variable to only hospitalisations after the age of 3 years with a diagnosis code of asthma.

\section{Statistical analysis}

We used Cox regression to estimate hazard ratios (HRs) where the outcome was time to first respiratory hospitalisation after the age of 3 years and the primary predictor was hospitalisation for ARI in the first year of life. Initial models included the primary predictor as a dichotomous variable (any ARI admission in the first year of life versus no admission). Further models then examined the number of ARI admissions $(0,1,2$ or $\geqslant 3)$ in the first year of life as a categorical variable. Our sensitivity analyses included four models with the different combinations of exposure and outcome. Maternal smoking during pregnancy was not recorded in 1996 so those births were removed from the analysis $(n=24671)$, as were those who died before the age of 3 years $(n=1133)$ and those births after 2002 that did not reach the age of 3 years at the end of the study period (December 31, 2005). Therefore, 145580 children were available for analysis. The time variable for the Cox regression was calculated as the minimum of time to first respiratory morbidity hospitalisation, time to death or time to end of the study period, after the age of 3 years. As the end of the study period was the end of 2005, the maximum age of children at the end of follow-up was 10 years, with the median follow-up time being 6.5 years.

Models were adjusted for sex, gestational age (categorised as <32 weeks, 32-36 weeks and >36 weeks), maternal asthma during pregnancy, maternal smoking during pregnancy, number of previous pregnancies, maternal age, mode of delivery, season of birth, Aboriginal status, presence of a major birth defect, percentage of optimal birthweight, SEIFA quantiles and infant's year of birth as a continuous variable. Further models were stratified according to gestational age. If any records had missing data for these covariates, they were dropped from the fully adjusted models. Effect modification was assessed by interaction models with a priori selected predictors and the primary predictor of number of ARI admissions in infancy. Likelihood ratio (LR) tests were conducted to determine whether interaction models with the interaction terms, main effects and all other confounding factors were a better fit to the data than the models with only main effects and confounding factors. As a marker of severity, total length of stay in hospital in the first year of life for ARI was included in the model as a continuous variable on a log scale. Admissions for ARI were then segregated into those that had a length of stay $\geqslant 3$ days (severe ARI) and those that stayed in hospital $<3$ days (less severe ARI). We present unadjusted and adjusted hazard ratios and $95 \%$ confidence intervals for each of the Cox models using robust standard errors. The proportionality assumption in the final models was tested by assessing log-log plots to visually assess whether the curves were parallel and formally tested using Schoenfeld residuals. Using the punafcc function in STATA (StataCorp, College Station, TX, USA), we calculated the population attributable fraction and 95\% confidence intervals for selected predictor variables in our final models. All data cleaning and analyses were conducted in IBM SPSS Statistics (version 22; Armonk, NY, USA) and STATA version 13.

\section{Results}

From the cohort of 145580 children available for analysis, 7911 (5.4\%) had at least one ARI hospitalisation in the first year of life with a median length of stay of 3 days. For those that had at least one ARI hospitalisation, the median number of ARI admissions in the first year of life was 1 (mean 1.33, range 1-17). The most common principal diagnosis in the ARI category was bronchiolitis (62\%) followed by pneumonia (10\%). Slightly fewer than 3000 children $(2.1 \%)$ had a hospitalisation for respiratory causes after the age of 3 years. The most common principal diagnosis after the age of 3 years was asthma (71\%), followed by unspecified acute lower respiratory infection (11\%). The composition of the ARI and respiratory hospitalisation categories did not change over the duration of the study (data not shown).

Hospitalisation for ARI in the first 12 months of life was significantly associated with respiratory hospitalisation after the age of 3 years, before and after adjusting for known perinatal risk factors (HR 3.5, 95\% CI 3.1-3.8; adjusted HR 3.0, 95\% CI 2.6-3.4). 27\% of observations had missing covariate data and were consequently dropped from the fully adjusted models. The magnitude of the hazard ratio for ARI was significantly larger than the hazard ratios for all the other risk factors (table 1). There was a dose-response effect with the number of ARI hospitalisations in the first year of life (fig. 1 and table 2) in the direction of a stronger risk of respiratory hospitalisation after 3 years with increasing number of ARI admissions in infancy.

Restricting the exposure to infant hospitalisations coded for acute lower respiratory infections only (removing asthma and wheeze) did not change the hazard ratios (table 3). Restricting the outcome to hospitalisations after the age of 3 years coded for asthma only slightly lowered the magnitude of the 
TABLE 1 Hospitalisations for acute respiratory infection (ARI) before the age of 12 months, maternal and infant characteristics and subsequent risk of respiratory morbidity after the age of 3 years

\begin{tabular}{|c|c|c|c|c|}
\hline & \multicolumn{2}{|c|}{$\begin{array}{c}\text { Respiratory morbidity } \\
\geqslant 3 \text { years }\end{array}$} & \multirow[t]{2}{*}{ Unadjusted } & \multirow[t]{2}{*}{ Adjusted } \\
\hline & Yes & No & & \\
\hline \multicolumn{5}{|l|}{ ARI $<12$ months } \\
\hline Yes & $484(6.1)$ & 7427 (93.9) & $3.46(3.14-3.81)$ & $2.97(2.62-3.37)$ \\
\hline No & $2500(1.8)$ & 135169 (98.2) & Ref. & Ref. \\
\hline \multicolumn{5}{|l|}{ Sex } \\
\hline Male & $1806(2.4)$ & $72611(97.6)$ & 1.47 (1.37-1.58) & $1.33(1.22-1.45)$ \\
\hline Female & $1178(1.6)$ & 69985 (98.3) & Ref. & Ref. \\
\hline \multicolumn{5}{|l|}{ Gestational age weeks } \\
\hline$<32$ & $69(6.5)$ & 988 (93.5) & $3.28(2.58-4.17)$ & $2.19(1.62-2.98)$ \\
\hline $32-36$ & $208(2.5)$ & 8074 (97.5) & $1.28(1.11-1.48)$ & $1.03(0.86-1.23)$ \\
\hline$>36$ & $2691(2.0)$ & $132587(98.0)$ & Ref. & Ref. \\
\hline \multicolumn{5}{|l|}{ Maternal asthma } \\
\hline Yes & $357(2.8)$ & 12563 (97.2) & $1.56(1.40-1.74)$ & $1.43(1.27-1.62)$ \\
\hline No & $2623(2.0)$ & 129606 (98.0) & Ref. & Ref. \\
\hline \multicolumn{5}{|l|}{ Maternal smoking } \\
\hline Yes & $643(2.3)$ & 27063 (97.7) & $1.26(1.15-1.38)$ & $1.05(0.94-1.17)$ \\
\hline No & $1810(1.8)$ & 98464 (98.2) & Ref. & Ref. \\
\hline \multicolumn{5}{|l|}{ Maternal age years } \\
\hline$<20$ & $227(2.7)$ & 8243 (97.3) & 1.35 (1.15-1.59) & $1.07(0.85-1.35)$ \\
\hline $20-24$ & 585 (2.3) & $24152(97.7)$ & $1.15(1.02-1.31)$ & $1.05(0.89-1.24)$ \\
\hline $25-29$ & $933(2.1)$ & 44155 (97.9) & 1.02 (0.91-1.14) & $0.96(0.83-1.10)$ \\
\hline $30-34$ & $793(1.8)$ & $43184(98.2)$ & $0.91(0.81-1.03)$ & $0.92(0.80-1.06)$ \\
\hline$\geqslant 35$ & $445(2.0)$ & $22400(98.1)$ & Ref. & Ref. \\
\hline \multicolumn{5}{|l|}{ Previous pregnancies $\mathrm{n}$} \\
\hline 0 & $845(2.0)$ & 41045 (98.0) & Ref. & Ref. \\
\hline 1 & $853(1.9)$ & $43858(98.1)$ & $0.94(0.86-1.04)$ & $0.95(0.84-1.07)$ \\
\hline 2 & $572(2.1)$ & $27373(98.0)$ & $1.01(0.91-1.13)$ & $1.06(0.93-1.22)$ \\
\hline 3 & $710(2.3)$ & $29893(97.7)$ & $1.14(1.04-1.27)$ & $1.06(0.92-1.22)$ \\
\hline \multicolumn{5}{|l|}{ Mode of delivery } \\
\hline Vaginal & $1829(2.0)$ & 88052 (98.0) & Ref. & Ref. \\
\hline Instrumental & $383(2.0)$ & 18998 (98.2) & $0.96(0.86-1.07)$ & $1.02(0.88-1.17)$ \\
\hline Elective Caesarean & $408(2.0)$ & 19708 (98.0) & $1.06(0.95-1.18)$ & $1.08(0.95-1.23)$ \\
\hline Emergency Caesarean & $360(2.3)$ & $15411(97.7)$ & $1.19(1.07-1.34)$ & $1.14(0.99-1.31)$ \\
\hline \multicolumn{5}{|c|}{ Percentage of optimal birthweight } \\
\hline Low $<85 \%$ & $385(2.6)$ & $14510(97.4)$ & $1.29(1.16-1.44)$ & $1.07(0.94-1.23)$ \\
\hline Normal $85-114 \%$ & $2127(2.0)$ & 104633 (98.0) & Ref. & Ref. \\
\hline High $>114 \%$ & $277(2.1)$ & 12980 (97.9) & 1.06 (0.93-1.19) & $1.01(0.88-1.17)$ \\
\hline \multicolumn{5}{|l|}{ SEIFA quantile $\#$} \\
\hline $91-100 \%$ & $150(1.6)$ & $9419(98.4)$ & Ref. & Ref. \\
\hline $76-90 \%$ & $329(1.8)$ & $17568(98.2)$ & $1.17(0.96-1.42)$ & $1.15(0.92-1.44)$ \\
\hline $26-75 \%$ & $1280(2.0)$ & 63601 (98.0) & $1.23(1.04-1.45)$ & $1.23(1.01-1.51)$ \\
\hline $11-25 \%$ & $484(2.1)$ & 22560 (97.9) & $1.33(1.11-1.60)$ & $1.19(0.95-1.48)$ \\
\hline $0-10 \%$ & $370(2.7)$ & $13586(97.4)$ & $1.69(1.39-2.04)$ & $1.36(1.08-1.72)$ \\
\hline \multicolumn{5}{|l|}{ Season of birth } \\
\hline Summer & $698(2.0)$ & 34748 (98.0) & 1.05 (0.95-1.17) & $1.02(0.90-1.17)$ \\
\hline Autumn & $882(2.4)$ & $36374(97.6)$ & $1.23(1.11-1.37)$ & $1.17(1.03-1.33)$ \\
\hline Winter & $794(2.2)$ & 35505 (97.8) & $1.21(1.09-1.35)$ & $1.13(1.00-1.29)$ \\
\hline Spring & $610(1.7)$ & 35969 (98.3) & Ref. & Ref. \\
\hline \multicolumn{5}{|l|}{ Aboriginal status } \\
\hline Aboriginal & 341 (3.3) & 10059 (96.7) & $1.73(1.54-1.93)$ & $1.18(1.00-1.39)$ \\
\hline Non-Aboriginal & $2643(2.0)$ & $132467(98.0)$ & Ref. & Ref. \\
\hline \multicolumn{5}{|l|}{ Birth defect } \\
\hline Yes & $290(3.6)$ & $7859(96.4)$ & $1.79(1.58-2.02)$ & $1.48(1.27-1.73)$ \\
\hline No & $2694(2.0)$ & 134737 (98.0) & Ref. & Ref. \\
\hline Year of infant's birth & & & $1.07(1.04-1.10)$ & $1.08(1.03-1.12)$ \\
\hline \multicolumn{5}{|c|}{$\begin{array}{l}\text { Data are presented as } n(\%) \text { or hazard ratio }(95 \% \mathrm{Cl}) \text {, unless otherwise stated. SEIFA: Socio-Economic } \\
\text { Index for Area; Ref.: reference. \#: } 91-100 \% \text { represents those that are least disadvantaged while } 0-10 \% \\
\text { represents those that are the most disadvantaged; }{ }^{9} \text { : modelled as a continuous variable. }\end{array}$} \\
\hline
\end{tabular}




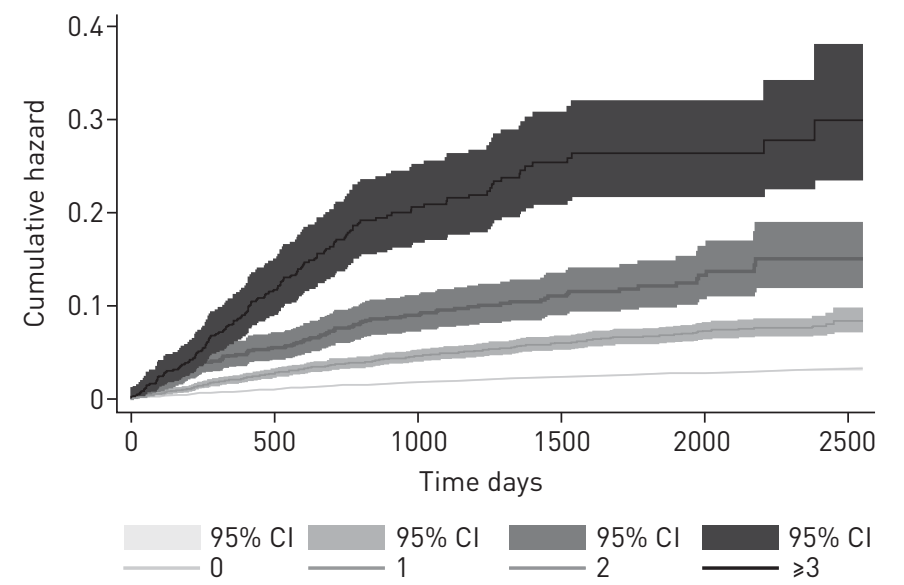

FIGURE 1 Cumulative hazard function of respiratory hospitalisation after the age of 3 years for increasing number of hospitalisations for acute respiratory infection before the age of 12 months.

hazard ratios, but did not result in significant changes (table 3). A similar phenomenon was noticed when the restricted exposure was assessed as a categorical variable to assess the dose-response relationship (data not shown). As the hazard ratios did not alter significantly, we used the larger ARI exposure category and hospitalisation for respiratory causes outcome category for all further models.

To assess whether the relationship between early ARI and subsequent respiratory hospitalisation risk was modified by infant or maternal characteristics, interactions with selected predictors and the number of ARI hospitalisations in infancy were included one at time into the model. The model did not show any effect modification with gestational age category (LR Chi-squared 6 degrees of freedom 4.30, p=0.64), mode of delivery (LR Chi-squared 9 degrees of freedom 6.49, $\mathrm{p}=0.69$ ), season of birth (LR Chi-squared 9 degrees of freedom 2.47, $\mathrm{p}=0.98$ ) and maternal smoking during pregnancy (LR Chi-squared 3 degrees of freedom 2.56, $\mathrm{p}=0.46$ ). Table 3 presents a stratified analysis across gestational age groups. The doseresponse relationship between risk of respiratory hospitalisation after the age of 3 years and recurrent ARI in infancy remained in all gestational age categories (table 4).

We further examined the effect of severity of ARI by investigating the total length of hospital stay for ARI in infancy. Including this in the model on a continuous log scale reduced the magnitude of the hazard ratios for frequency of ARI hospitalisations, but significantly improved the model (LR Chi-squared 1 degree of freedom 9.52, $\mathrm{p}=0.002$; online supplementary table $\mathrm{S} 1$, model 1 ) indicating that the longer a child stays in hospital, the stronger the risk of later respiratory morbidity. The model was not significantly improved when recurrent ARI hospitalisations were separated into severe and less severe (LR Chi-squared 3 degrees of freedom 4.46, $\mathrm{p}=0.22$; online supplementary table S1, model 2). The population attributable fraction of ARI in infancy was calculated as $10.7 \%$ (95\% CI 10.0-11.4\%), significantly higher than other risk factors (e.g. maternal smoking $1.2(-1.5-3.8) \%$; or <32 weeks gestation $1.3(0.9-1.6) \%)$. Log-log plots were assessed for all the final models and parallel curves for all levels of the primary predictor variable were observed $(\mathrm{p}>0.1)$, indicating that the proportionality assumption was upheld.

TABLE 2 Number of hospitalisations for acute respiratory infection before the age of 12 months and subsequent risk of respiratory hospitalisation after the age of 3 years

\begin{tabular}{|c|c|c|c|}
\hline \multicolumn{2}{|c|}{$\begin{array}{c}\text { Respiratory hospitalisation } \\
\geqslant 3 \text { years }\end{array}$} & \multirow[t]{2}{*}{ Unadjusted } & \multirow[t]{2}{*}{ Adjusted } \\
\hline Yes & No & & \\
\hline 2500 (1.8) & $135169(98.2)$ & Ref. & Ref. \\
\hline $296(4.7)$ & 5990 (95.3) & $2.65(2.35-2.99)$ & $2.47(2.13-2.87)$ \\
\hline $95(8.6)$ & 1006 (91.4) & $4.91(4.00-6.04)$ & $3.67(2.78-4.84)$ \\
\hline 93 (17.8) & 431 (82.2) & $10.48(8.51-12.90)$ & 8.57 (6.51-11.28) \\
\hline
\end{tabular}

Data are presented as $\mathrm{n}(\%)$ or hazard ratio $(95 \% \mathrm{CI})$, unless otherwise stated. Hazard ratios adjusted for sex, gestational age, maternal asthma during pregnancy, maternal smoking during pregnancy, mode of delivery, maternal age, number of previous pregnancies, percentage of optimal birthweight, Socio-Economic Index for Area quantile, season of birth, Aboriginal status, presence of any birth defect and year of infant's birth. Ref.: reference. 
TABLE 3 Sensitivity analysis of exposure and outcome measures

\begin{tabular}{|c|c|c|c|c|c|c|}
\hline & \multicolumn{2}{|c|}{ Exposure (<12 months) } & \multicolumn{2}{|c|}{ Outcome ( $\geqslant 3$ years) } & Unadjusted & Adjusted \\
\hline $\begin{array}{l}\text { Model 1: full model as per } \\
\text { tables } 1 \text { and } 2\end{array}$ & ARI & 12385 & $\begin{array}{c}\text { Respiratory } \\
\text { hospitalisation }\end{array}$ & 3775 & $3.46(3.14-3.81)$ & $2.97(2.62-3.37)$ \\
\hline Model 2: restricted exposure & $\begin{array}{l}\text { Respiratory } \\
\text { infection only }\end{array}$ & 11504 & $\begin{array}{c}\text { Respiratory } \\
\text { hospitalisation }\end{array}$ & 3775 & $3.48(3.15-3.84)$ & $2.97(2.60-3.38)$ \\
\hline Model 3: restricted outcome & ARI & 12385 & Asthma only & 3121 & $3.13(2.81-3.50)$ & $2.92(2.54-3.38)$ \\
\hline
\end{tabular}

Data are presented as $\mathrm{n}$ or hazard ratio $(95 \% \mathrm{CI})$, unless otherwise stated. Hazard ratios adjusted for sex, gestational age, maternal asthma during pregnancy, maternal smoking during pregnancy, mode of delivery, maternal age, number of previous pregnancies, percentage of optimal birthweight, Socio-Economic Index for Area quantile, season of birth, Aboriginal status, presence of any birth defect and year of birth of infant. ARI: acute respiratory infection.

\section{Discussion}

In a population-based cohort of children, we have shown that the strongest predictor of respiratory morbidity requiring hospitalisation after the age of 3 years is having one or more hospitalisations for ARI during infancy. This relationship remains after adjusting for several known risk factors for respiratory morbidity and is not modified by gestational age, maternal smoking during pregnancy, season of birth or mode of delivery. We also noted a clear dose response in that the more admissions for ARI in infancy, the stronger the association with hospitalisation for respiratory causes after 3 years.

We did not see any effect modification of a priori selected risk factors and the relationship of early ARI and later respiratory hospitalisations. Previous reports have suggested that infants with a predisposition to early viral infection, such as being born premature, are at a higher risk of recurrent severe respiratory infections requiring hospitalisation and later development of asthma [12, 26]. For confirmation of this association, we would expect to see some effect modification by gestational age, whereby those infants born prematurely would have a higher risk of later respiratory hospitalisation given early respiratory morbidity. In this analysis the overlapping hazard ratios across the various gestational age categories suggests the relationship of early

TABLE 4 Number of hospitalisations for acute respiratory infection before the age of 12 months and subsequent risk of respiratory hospitalisation after age 3 years in separate models according to gestational age

\begin{tabular}{|c|c|c|c|}
\hline & \multicolumn{2}{|c|}{$\begin{array}{c}\text { Respiratory } \\
\text { hospitalisation } \geqslant 3 \text { years }\end{array}$} & \multirow[t]{2}{*}{ Adjusted } \\
\hline & Yes & No & \\
\hline \multicolumn{4}{|c|}{ Model 1: gestational age $<32$ weeks } \\
\hline 0 & $34(4.3)$ & 749 (95.7) & Ref. \\
\hline 1 & $14(8.1)$ & 158 (91.9) & $1.74(0.76-3.98)$ \\
\hline 2 & 7 (12.5) & 49 (87.5) & $2.14(0.58-7.91)$ \\
\hline$\geqslant 3$ & $14(30.4)$ & $32(69.6)$ & $6.89(2.72-17.43)$ \\
\hline \multicolumn{4}{|c|}{ Model 2: gestational age 32-36 weeks } \\
\hline 0 & $153(2.1)$ & 7277 (97.9) & Ref. \\
\hline 1 & $31(4.9)$ & $596(95.1)$ & $2.11(1.27-3.49)$ \\
\hline 2 & $11(7.7)$ & 132 (92.3) & $2.77(1.17-6.52)$ \\
\hline$\geqslant 3$ & $13(15.9)$ & $69(84.2)$ & $7.34(3.30-16.32)$ \\
\hline \multicolumn{4}{|c|}{ Model 3: gestational age $>36$ weeks } \\
\hline 0 & $2301(1.8)$ & $126291(98.2)$ & Ref. \\
\hline 1 & $249(4.6)$ & $5176(95.4)$ & $2.54(2.17-2.98)$ \\
\hline 2 & 77 (8.7) & 805 (91.3) & $3.97(2.95-5.34)$ \\
\hline$\geqslant 3$ & $64(16.9)$ & $315(83.1)$ & $8.94(6.50-12.29)$ \\
\hline \multicolumn{4}{|c|}{$\begin{array}{l}\text { Data are presented as } \mathrm{n}(\%) \text { or hazard ratio }(95 \% \mathrm{CI}) \text {, unless otherwise stated. Hazard ratios adjusted for sex, } \\
\text { maternal asthma during pregnancy, maternal smoking during pregnancy, mode of delivery, maternal age, } \\
\text { number of previous pregnancies, percentage of optimal birthweight, Socio-Economic Index for Area score, } \\
\text { season of birth, Aboriginal status, presence of any birth defect and year of infant's birth. Ref.: reference. }\end{array}$} \\
\hline
\end{tabular}


and recurrent ARI with increased risk of later respiratory hospitalisation and asthma is similar in preterm infants and late term infants. Montgomery et al. [27] conducted a similar analysis of a large Swedish population cohort. The authors did report effect modification with gestational age with the risk of subsequent asthma after 5 years, but only in those born at $<28$ weeks gestation and they did not explore recurrent ARI in infancy.

Previous studies have focused on a single pathogen such as respiratory syncytial virus (RSV) [9] or rhinovirus [28] or a specific clinical condition, such as bronchiolitis, and the relationship with subsequent asthma. However, in our previous analyses of linked administrative laboratory and hospitalisation data, we have shown that in the first 2 years of life, various respiratory pathogens are detected across a range of ARI diagnostic categories and not all possible causative pathogens, such as rhinovirus, are routinely tested for [29]. In addition, for the purpose of quantifying the risk of early respiratory infection on later respiratory hospitalisations and identifying potential effect modifiers in that relationship, the causative viral pathogen of the early infection is not of critical importance, as the clinical presentation of respiratory viruses in infancy is likely to be similar. Therefore, it is perhaps more useful to look at a combination of conditions in the first 12 months of life to indicate ARI, which is a strength of our analysis.

We did not have data on some additional known risk factors for later respiratory hospitalisations such as childcare attendance, duration of breastfeeding, family history of asthma and allergies and immunisation status, which is a limitation to our study. In addition, we currently do not have access to any data at the primary healthcare level on ARI and respiratory morbidity episodes not requiring hospitalisation, therefore our findings are based on the severe end of the clinical spectrum in terms of respiratory morbidity. Nonetheless, using longitudinal population-based data we have shown an independent significant dose response in the frequency of hospitalisation for ARI in infancy and risk of later respiratory morbidity and asthma requiring hospitalisation. There is growing debate around whether the relationship between early respiratory infection, particularly viral infection, and later development of respiratory morbidity or asthma is causal [12]. Causation has been demonstrated in mouse models with long-term impairment of lung function following early influenza infection [30]. Our retrospective observational study cannot demonstrate causation, rather the association between the exposure and outcome, where in this case, exposure may be just early signs of the disease (or its exacerbations). However, to assist in the reduction of later respiratory hospitalisations and asthma in childhood, attention should be focused on modifiable and preventable risk factors to target the recurrence of early ARI. Our earlier work reported on population-attributable fractions to hospitalisations for acute lower respiratory infection in children before the age of 2 years using this same dataset [4]. We identified some modifiable factors, such as elective Caeasarean, maternal smoking in pregnancy and teenage pregnancy, that could be targeted for public health campaigns. We also identified factors that in isolation are not easily modifiable, such as male sex, autumn-born babies and multiple previous pregnancies, but highlight susceptible subgroups to specifically target for disease intervention measures such as increased hand hygiene measures, timeliness of standard vaccinations and passive immunisation. In this current analysis, we have shown that the population-attributable fraction of ARI in infancy for later respiratory hospitalisation is $\sim 11 \%$, far greater than for other modifiable factors such as maternal smoking in pregnancy and preterm birth $(\sim 1 \%)$.

We have shown that RSV is the most commonly identified pathogen found in children hospitalised with respiratory infections in infancy [29]. Prophlyaxis with RSV monoclonal antibody, palivizumab, administered as monthly intramuscular infections, has been shown to be effective in reducing RSV-confirmed hospitalisations in high-risk populations and a reduction in wheezing days during the first year of life [31-33]. We have shown here that length of stay in hospital during infancy was a significant predictor to later respiratory morbidity, indicating that the more severe the initial ARI, the stronger the risk of later respiratory morbidity requiring hospitalisation. While this adds to the evidence that severity of early ARI may have an impact on later lung development [12], it also highlights the importance of reducing severity of ARI in the first year of life. Further studies are now needed to potentially understand the full effect of prophylaxis on preventing respiratory morbidity.

In conclusion, using population-based longitudinal data we have shown that the strongest predictor of respiratory morbidity and asthma requiring hospitalisation after 3 years of age is recurrent hospitalisation for ARI in infancy regardless of gestational age, season of birth, mode of delivery and maternal smoking during pregnancy. While these factors do not impact on the relationship between early infection and later morbidity, they are known risk factors for early infection. If prevention efforts are focused on early infection, the benefit is likely to extend to later morbidity.

\section{Acknowledgements}

We would to acknowledge the ongoing support from the Infectious Diseases Community Reference Group at the Telethon Kids Institute (Perth, Australia). The use of linked data was approved by the Western Australia Data Linkage Branch at the Western Australian Department of Health. 


\section{References}

1 Deshpande SA, Northern V. The clinical and health economic burden of respiratory syncytial virus disease among children under 2 years of age in a defined geographical area. Arch Dis Child 2003; 88: 1065-1069.

2 Peck AJ, Holman RC, Curns AT, et al. Lower respiratory tract infections among American Indian and Alaska Native children and the general population of U.S. children. Pediatr Infect Dis J 2005; 24: 342-351.

3 O'Grady KA, Torzillo PJ, Chang AB. Hospitalisation of Indigenous children in the Northern Territory for lower respiratory illness in the first year of life. Med J Aust 2010; 192: 586-590.

4 Moore HC, de Klerk N, Richmond P, et al. A retrospective population-based cohort study identifying target areas for prevention of acute lower respiratory infections in children. BMC Public Health 2010; 10: 757.

5 Zachariah P, Ruttenber M, Simões EAF. Hospitalizations due to respiratory syncytial virus in children with congenital malformations. Pediatr Infect Dis J 2011; 30: 442-445.

6 Jackson DJ, Gangnon RE, Evans MD, et al. Wheezing rhinovirus illnesses in early life predict asthma development in high-risk children. Am J Respir Crit Care Med 2008; 178: 667-672.

7 Kusel MM, de Klerk NH, Kebadze T, et al. Early-life respiratory viral infections, atopic sensitization, and risk of subsequent development of persistent asthma. J Allergy Clin Immunol 2007; 119: 1105-1110.

8 Sigurs N, Aljassim F, Kjellman B, et al. Asthma and allergy patterns over 18 years after severe RSV bronchiolitis in the first year of life. Thorax 2010; 65: 1045-1052.

9 Sigurs N, Gustafsson PM, Bjarnason R, et al. Severe respiratory syncytial virus bronchiolitis in infancy and asthma and allergy at age 13. Am J Respir Crit Care Med 2005; 171: 137-141.

10 Stensballe LG, Simonsen JB, Thomsen SF, et al. The causal direction in the association between respiratory syncytial virus hospitalization and asthma. J Allergy Clin Immunol 2009; 123: 131-137.

11 Young S, O'Keeffe PT, Arnott J, et al. Lung function, airway responsiveness, and respiratory symptoms before and after bronchiolitis. Arch Dis Child 1995; 72: 16-24.

12 Mackenzie KJ, Anderton SM, Schwarze J. Viral respiratory tract infections and asthma in early life: cause and effect? Clin Exp Allergy 2014; 44: 9-19.

13 Kristensen K, Fisker N, Haerskjold A, et al. Caesarean section and hospitalization for respiratory syncytial virus infection: a population-based study. Pediatr Infect Dis J 2015; 34: 145-148.

14 Moore HC, de Klerk N, Holt P, et al. Hospitalisation for bronchiolitis in infants is more common after elective caesarean delivery. Arch Dis Child 2012; 97: 410-414.

15 Thavagnanam S, Fleming J, Bromley A, et al. A meta-analysis of the association between Caesarean section and childhood asthma. Clin Exp Allergy 2008; 38: 629-633.

16 Been JV, Lugtenberg MJ, Smets E, et al. Preterm birth and childhood wheezing disorders: a systematic review and meta-analysis. PLoS Med 2014; 11: e1001596.

17 Vrijlandt EJ, Kerstjens JM, Duiverman EJ, et al. Moderately preterm children have more respiratory problems during their first 5 years of life than children born full term. Am J Respir Crit Care Med 2013; 187: 1234-1240.

18 Holman CD, Bass AJ, Rosman DL, et al. A decade of data linkage in Western Australia: strategic design, applications and benefits of the WA data linkage system. Aust Health Rev 2008; 32: 766-777.

19 Moore HC, Lehmann D, de Klerk N, et al. Reduction in disparity for pneumonia hospitalisations between Australian Indigenous and non-Indigenous children. J Epidemiol Community Health 2012; 66: 489-494.

20 Blair EM, Liu Y, de Klerk NH, et al. Optimal fetal growth for the Caucasian singleton and assessment of appropriateness of fetal growth: an analysis of a total population perinatal database. BMC Pediatr 2005; 5: 13 .

21 McLennan W. 1996 Census of Population and Housing: Socio-economic Indexes for Areas. Canberra, Australian Bureau of Statistics, 1998.

22 Jama-Alol KA, Moore HC, Jacoby P, et al. Morbidity due to acute lower respiratory infection in children with birth defects: a total population-based linked data study. BMC Pediatr 2014; 14: 80.

23 Moore $\mathrm{H}$, Burgner D, Carville $\mathrm{K}$, et al. Diverging trends for lower respiratory infections in non-Aboriginal and Aboriginal children. J Paediatr Child Health 2007; 43: 451-457.

24 Björ O, Bråbäck L. A retrospective population based trend analysis on hospital admissions for lower respiratory illness among Swedish children from 1987 to 2000. BMC Public Health 2003; 3: 22.

25 van Woensel JB, van Aalderen WM, Kneyber MC, et al. Bronchiolitis hospitalisations in the Netherlands from 1991 to 1999. Arch Dis Child 2002; 86: 370-371.

26 Drysdale SB, Wilson $\mathrm{T}$, Alcazar $\mathrm{M}$, et al. Lung function prior to viral lower respiratory tract infections in prematurely born infants. Thorax 2011; 66: 468-473.

27 Montgomery S, Bahmanyar S, Brus $\mathrm{O}$, et al. Respiratory infections in preterm infants and subsequent asthma: a cohort study. BMJ Open 2013; 3: e004034.

28 O'Callaghan-Gordo C, Bassat Q, Díez-Padrisa N, et al. Lower respiratory tract infections associated with rhinovirus during infancy and increased risk of wheezing during childhood. A cohort study. PLoS One 2013; 8: e69370.

29 Moore HC, De Klerk N, Keil AD, et al. Use of data linkage to investigate the aetiology of acute lower respiratory infection hospitalisations in children. J Paediatr Child Health 2012; 48: 520-528.

30 Larcombe AN, Foong RE, Bozanich EM, et al. Sexual dimorphism in lung function responses to acute influenza A infection. Influenza Other Respir Viruses 2011; 5: 334-342.

31 Feltes TF, Cabalka AK, Meissner HC, et al. Palivizumab prophylaxis reduces hospitalization due to respiratory syncytial virus in young children with hemodynamically significant congenital heart disease. J Pediatr 2003; 143: 532-540.

32 The IMpact-RSV Study Group. Palivizumab, a humanized respiratory syncytial virus monoclonal antibody, reduces hospitalization from respiratory syncytial virus infection in high-risk infants. Pediatrics 1998; 102: 531-537.

33 Blanken MO, Rovers MM, Molenaar JM, et al. Respiratory syncytial virus and recurrent wheeze in healthy preterm infants. N Engl J Med 2013; 368: 1791-1799. 